\title{
Eine gemeinsame Entscheidung
}

\section{Anne-Françoise Allaz}

Prof. Dr. med., Mitglied der Redaktion

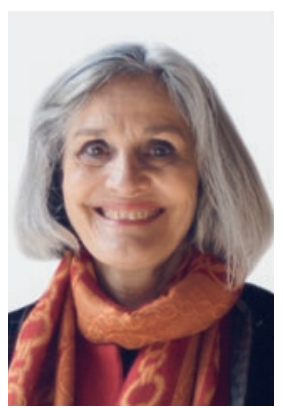

Ein Freund ruft mich an, sehr aufgewühlt. Seine Frau leidet an Krebs im fortgeschrittenen Stadium und ist gerade ins Spital eingeliefert worden. Sehr angenehme Aufnahme, kompetente Behandlung, vertrauenswürdiges Team. "Aber warum schauen all diese Ärzte nacheinander vorbei und wollen von meiner Frau wissen, mit welchen medizinischen Massnahmen sie einverstanden ist? Das beunruhigt sie enorm.» Ich erläutere, dass die Spitäler (ebenso wie die Ärztinnen und Ärzte) heute verpflichtet sind, die Patientinnen und Patienten über deren Gesundheitszustand zu informieren, sich an deren Patientenverfügungen zu orientieren und deren Entscheidungen zu respektieren. Diese Entscheidungen müssen den an der Behandlung beteiligten Teams klar mitgeteilt werden.

Ich kenne diese Erklärung auswendig, die ich angesichts der überraschten oder gar bestürzten Reaktionen von Patientinnen und Patienten oder Angehörigen schon oft geben musste. Ich stehe zum Grundgedanken dieser Regelung, ihre Anwendung aber bringt mich ins Grübeln. In den letzten vier Jahrzehnten hat sich die Kommunikation mit den Patientinnen und Patienten tiefgreifend verändert - von einer eher paternalistischen Haltung hin zu einer umfassenden Informierung und gemeinsamen Entscheidungsfindung, dem shared decision-making. Diese gesellschaftliche Entwicklung, sich auf die «informierten Präferenzen» und Werte der Patientinnen und Patienten und nicht allein auf medizinische Aspekte zu stützen, ist eine Reaktion auf die Forderung nach der Einbindung von Patientinnen und Patienten in ihre Gesundheitsversorgung. Zusammenfassen lässt sich das im Slogan nothing about me, without me, ever. Heute scheinen der Zugang der Patientinnen und Patienten zu ihrer Krankengeschichte und eine gemeinsame Entscheidungsfindung in der medizinischen Ethik und Kultur unserer Breitengrade selbstverständlich. Dies geht einher mit steigenden medizinisch-rechtlichen Anforderungen an die Information der Patientinnen und Patienten, die Ermittlung der von ihnen bevorzugten medizinischen Massnahmen und die Dokumentation. Aber was sagen die betroffenen Patientinnen und Patienten dazu?

Die Frau meines Freundes bleibt aufgewühlt. Das Fachpersonal will unbedingt die gewünschten Massnahmen besprechen. So weit ist sie noch nicht. Sie befindet sich im luftleeren Raum: Sie weiss, was sie hat, ohne es wirklich begriffen zu haben. Wie könnte man es besser ausdrücken? Ihr Wissen über ihren Zustand ist noch rein intellektuell, sie hat es weder verinnerlicht noch emotional akzeptiert. Eine Situation, der wir so oft begegnen! Manche sehen darin Verleugnung, andere Hoffnung. Man richtet sich in der Unentschlossenheit ein, die weniger beängstigend ist als eine klare Entscheidung, für die es noch viel zu früh zu sein scheint! «O Zeit, halt ein im Flug», deklamierte Alphonse de Lamartine. All dies erschwert den Ärztinnen und Ärzten, die gerne eine "Herangehensweise» festhalten würden, die Begleitung der Kranken; all dies macht der Patientin und ihren Angehörigen die Situation schwer erträglich, die hoffen, «Zeit gewinnen» zu können, indem sie eben noch keine «Entscheidung» treffen - auch keine gemeinsame.

Das in vielen Leitlinien festgelegte Eingehen auf die Bedürfnisse und Wünsche der Patientinnen und Patienten ist ein komplexes Thema. Ein individuelles Herangehen ist von grösster Bedeutung, schliesslich gilt: one size doesn't fit all. Die umfangreiche Literatur zur gemeinsamen Entscheidungsfindung zeigt, dass die Mehrheit der Patientinnen und Patienten zwar eine gemeinsame Entscheidung wünscht, andere sich von diesem medizinischen Ansatz jedoch überfordert fühlen. Aber auch diesen müssen wir gerecht werden.

Damit das hehre Ideal der gemeinsamen Entscheidungsfindung nicht unter die Räder einer strengen Normativität gerät, die Ziel und Nutzen untergraben könnte [1], weisen führende Köpfe darauf hin, dass die individuellen, kontextuellen und humanistischen Aspekte niemals hintenan gestellt werden dürfen [1, 2]. Es ist notwendig, die mit Krankheit und der Einlieferung ins Spital einhergehende Verletzlichkeit zu berücksichtigen; sich für den bisherigen Lebenslauf der Patientinnen und Patienten zu interessieren, der sie zu scheinbar irrationalen Entscheidungen führen kann; sich Zeit für ein «aktives Zuhören» zu nehmen, um der jeweiligen Person und ihren Werten Raum zu geben, ihr Respekt und Aufmerksamkeit zu schenken.

Kurz: Damit eine medizinische Entscheidung wirklich gemeinsam erfolgt, muss sie sich von einem standardisierten Modell lösen und das Risiko einer echten Begegnung eingehen. Dies erfordert Bereitschaft, die Fähigkeit zum Zuhören und Einfühlungsvermögen, wird aber bei den Beteiligten sicher für ein deutlich besseres Gefühl sorgen. 